\title{
Conductive junctions with parallel graphene sheets
}

\author{
Xiao Zheng, ${ }^{1}$ San-Huang $\mathrm{Ke}^{2,1, \mathrm{a})}$ and Weitao Yang ${ }^{1, \mathrm{~b})}$ \\ ${ }^{1}$ Department of Chemistry, Duke University, Durham, North Carolina 27708, USA \\ ${ }^{2}$ Department of Physics, Tongji University, 1239 Siping Road, Shanghai 200092, \\ People's Republic of China
}

(Received 10 December 2009; accepted 16 February 2010; published online 18 March 2010)

\begin{abstract}
The establishment of conductive graphene-molecule-graphene junction is investigated through first-principles electronic structure calculations and quantum transport calculations. The junction consists of a conjugated molecule connecting two parallel graphene sheets. The effects of molecular electronic states, structure relaxation, and molecule-graphene contact on the conductance of the junction are explored. A conductance as large as 0.38 conductance quantum is found achievable with an appropriately oriented dithiophene bridge. This work elucidates the designing principles of promising nanoelectronic devices based on conductive graphene-molecule-graphene junctions.
\end{abstract} (C) 2010 American Institute of Physics. [doi:10.1063/1.3357416]

\section{INTRODUCTION}

Since its first isolation achieved by Novoselov et al. ${ }^{1}$ in 2004, graphene, a single layer of graphite, has attracted increasing interest, due to its exceptional mechanical and electronic properties. ${ }^{2}$ This one-atom-thick material is a twodimensional (2D) semimetal. ${ }^{3}$ Its ballistic in-plane electron transport with zero electron effective mass has led to a variety of applications, such as single-electron transistors, ${ }^{4}$ single-molecule detection, ${ }^{5}$ spintronics, ${ }^{6}$ etc. Recently Bunch et $a l^{7}$ have demonstrated that, under controlled pressure, a suspended graphene sheet can extrude out by more than 100 $\mathrm{nm}$ without breaking. Therefore, by positioning two suspended graphene membranes back-to-back and bowing toward each other under pressure, technically it is now possible to make the gap between parallel graphene sheets within nanometer scale. This gap may possibly be filled by a bridging molecule which is covalently bonded to graphene sheets at its both ends. Such a new kind of graphene-moleculegraphene (GMG) junctions may exhibit unique physical properties and are potentially useful as electronic and optoelectronic devices. However, bulk graphene is only conductive in plane, but a junction with a bridging molecule is off plane. It is thus intriguing how to make such a GMG junction electronically conductive, so that based on it transport measurements can be realized. Thermodynamic stability is a fundamental issue for the construction of GMG junctions, as the planar $\mathrm{sp}^{2}$ network of bulk graphene is usually assumed to be inert due to the delocalized $\pi$-conjugation. Covalent functionalization of various allotropes of $\mathrm{sp}^{2}$ carbon such as graphene sheet and carbon nanotube has been achieved experimentally by the reduction of diazonium salts through either electrochemical ${ }^{8}$ or chemical grafting processes. ${ }^{9,10}$ Theoretical investigations ${ }^{11,12}$ have demonstrated that the chemical absorption of various types of functional groups on the graphene surface are thermally stable. The major techni-

\footnotetext{
a)Electronic mail: shke@tongji.edu.cn.

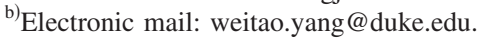

cal difficulty that remains is how to form covalent bonds between a bridging molecule and two parallel graphene sheets simultaneously. A possible solution is via a sequential bonding scheme based on techniques of manipulating graphene membranes. ${ }^{7}$ After functionalization of the first graphene sheet, a second sheet is properly positioned and its local geometry (and thus its free energy) is tuned through external mechanical suspension or pressure control, to facilitate formation of the second molecule-graphene bond. The present work will focus on unraveling the fundamental principles that govern the transport transparency of such a novel type of junctions.

Figure 1 depicts a prototypical GMG junction of our primary interest, where a dithiophene molecule is sandwiched between two parallel layers of pristine graphene. Each graphene layer extends to infinity in 2D ( $x y$-plane). At either side of the dithiophene bridge, the terminal carbon atom on dithiophene (referred to as link hereafter) is covalently bonded to its nearest neighbor on graphene (termed as anchor). The formation of a link-anchor bond will perturb the electronic $\pi$-conjugation originated from the $\mathrm{sp}^{2}$-carbon network and give rise to the local geometric deformation of originally planar graphene, as shown clearly in Fig. 1(a).

To investigate the transport properties of GMG junctions, first-principles calculations are carried out as follows.

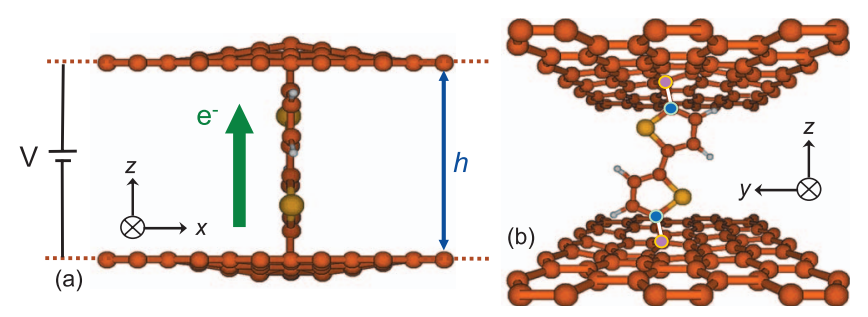

FIG. 1. A diagrammatic illustration of electron transport through a graphene-dithiophene-graphene junction. Both the graphene sheets extend to infinity in $x$ and $y$ directions. (a) Side view of the junction, with $h$ being the vertical separation between planar areas of two graphene layers. (b) Perspective view of the junction proximity, with the blue (red) circles representing the link (anchor) atoms of the bridging molecule (graphene). 
We first set up two ideal layers of graphene parallel to each other at a fixed separation $h$. A candidate bridging molecule is then positioned and oriented appropriately to make covalent contacts to the both graphene sheets. Then the bridging molecule together with the surrounding carbon atoms on graphene are subjected to geometric relaxation; while the structure of bulk graphene (sufficiently far away from anchor sites) remains intact, to mimic the suspended graphene used in a realistic experiment. The ground-state (zero-bias) conductance is subsequently calculated, based on which the relevant transport properties of presently studied GMG are analyzed and evaluated.

We employ standard density functional theory (DFT) for both geometric relaxation and conductance calculations, using a single zeta basis set for the Kohn-Sham wave functions, and the local-density approximation for exchangecorrelation functional. ${ }^{13}$ For the transport calculation, the nonequilibrium Green's function (NEGF) formalism ${ }^{14}$ is adopted to treat the open Kohn-Sham system. ${ }^{15}$ Specifically, the system is divided into three parts: top lead $(t)$, bottom lead $(b)$, and device region $(d)$. The latter contains the molecular bridge plus parts of the graphene sheets around the two ends to accommodate the molecule-graphene interaction. The self-consistent DFT Hamiltonian of the device region plus the self-energies of the two 2D graphene leads, $\boldsymbol{\Sigma}_{t / b}(\epsilon)$, are used to construct a single-particle Green's function, $\mathbf{G}_{d}(\boldsymbol{\epsilon})$, from which the transmission coefficient is calculated as a function of energy $T(\epsilon)=\operatorname{Tr}\left[\boldsymbol{\Gamma}_{t} \mathbf{G}_{d} \boldsymbol{\Gamma}_{b} \mathbf{G}_{d}^{\dagger}\right]$, where $\boldsymbol{\Gamma}_{t / b}(\epsilon)$ $=i\left[\boldsymbol{\Sigma}_{t / b}(\boldsymbol{\epsilon})-\boldsymbol{\Sigma}_{t / b}^{\dagger}(\boldsymbol{\epsilon})\right]$ is the coupling to the top $(t)$ or bottom (b) lead. Note that in the present case of the 2D leads, we calculate the self-energies by using the surface Green's function of the 2D graphene bulk as explained below.

Periodic boundary condition is imposed to account for the infinite 2D graphene with a unit cell consisting of 72 $(6 \times 12)$ carbon atoms in each layer, see Fig. 1. The graphene atoms within a radius of $4.5 \AA$ from the anchor sites are all relaxed, and the forces on those outside this range are found negligibly small. Therefore, further expansion of the relaxation area has rather minor effect on the outcome. The separation $h$ is kept larger than $6 \AA$ to avoid possible complication introduced to the NEGF formulation of transport current and electron distribution by any nontrivial van der Waals interactions between the two graphene sheets. The details of computational techniques for transport can be found in previous works. ${ }^{16}$ However, it is important to note that unlike in conventional quasi-one-dimensional transport scenario, in the GMG junction of our interest electrons move almost perpendicular to the $2 \mathrm{D}$ graphene planes when transmitting across the bridging molecule. The surface Green's function of the $2 \mathrm{D}$ bulk graphene is calculated by a fine sampling of the reciprocal space (see Appendix).

There are several well-known problems that plague the general DFT-NEGF approach for quantum transport calculations. (1) It is difficult to accurately determine the Fermi energy of the composite system of molecular device plus electrodes, particularly when the electrodes are made of different materials. (2) In the cases where the couplings between the device and electrodes are rather weak, the electron resonant transport plays a dominant role, and a "mean-field- like" Kohn-Sham scheme would become inadequate for describing the electronic response. ${ }^{16-18}$ (3) Conventional exchange-correlation functionals cannot accurately account for the possible significant charge transfer between the device and electrodes while they are brought into contact. ${ }^{19-21}$ However, these problems are absent from the DFT-NEGF calculations on the GMG junctions of our interest, and hence the results obtained are expected to be at least qualitatively accurate, due to the following facts. (1) The two electrodes are of same material, the bulk graphene sheet. The Fermi energy of a GMG junction is fixed at the Fermi energy of the bulk graphene electrodes. This is validated by our calculation results, where a sufficient amount of atoms on graphene is included in the device region to ensure the bulk properties, such as the distributions of electrostatic potential and electron density, are recovered at the device-electrode boundary. (2) For GMG junctions studied in this work, the molecular bridge is strongly coupled to graphene electrodes via covalent bonds, so that the resonance effect is negligible. (3) It is unlikely to have significant charge transfer in the GMG junctions considered in this work, as we focus on conjugated organic bridging molecules which are compatible to graphene sheet in terms of electronic structure.

Among the various types of bridging molecules examined, we find that thiophene oligomers evidently excel all the other candidates in terms of conductance. In the following, the key advantages of using the oligothiophenes (OTPs) are elaborated. Along the line, we explore the necessary conditions for making a conductive GMG junction.

\section{KEY FACTORS THAT AFFECT GMG JUNCTION CONDUCTANCE}

\section{A. Transport transparency of the bridging molecule}

Electrons can move ballistically in the bulk graphene, and the scattering processes, which degrade the junction conductance, occur mostly inside the bridging molecule or at the contacts. Linear $\pi$-conjugated molecules are thus promising candidates as conductive bridges since the delocalized $\pi$-orbitals may provide ballistic channels for the electron transmission.

We have considered various types of conjugated molecules, such as polyynes, benzene derivatives, OTPs, and phenylene-ethynylene oligomers (OPEs). Figure 2 depicts the calculated optimized structures and transport properties of four GMG junctions. As displayed in the left column of Fig. 2, all the bridging molecules are purposely tilted by a substantial angle from the vertical axis. Such inclined orientations are vital to achieve a nontrivial conductance across the junction, which will be elaborated later. The corresponding local density of states (LDOS) in the energy window of $\left(E_{\mathrm{f}}-0.5 \mathrm{eV}, E_{\mathrm{f}}+0.5 \mathrm{eV}\right)$ for each junction is displayed in the right column of Fig. 2, with $E_{\mathrm{f}}$ being the equilibrium Fermi energy of the entire composite system. The LDOS clearly confirms our expected scenario that the extended $\pi$-orbitals of the sandwiched molecule effectively "bridge" the $\pi$-conjugation on different layers of graphene. In the Landauer-Büttiker picture of quantum transport, ${ }^{22-24}$ the extended LDOS indicates the potential existence of conducting 

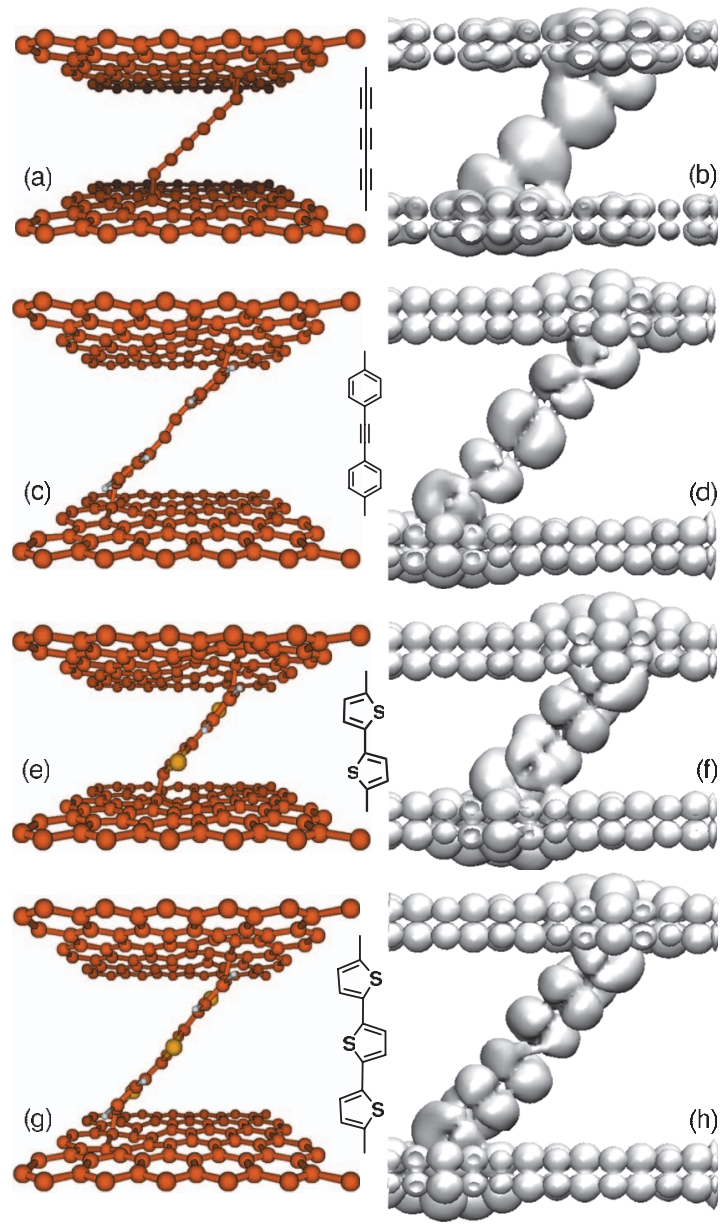

FIG. 2. Each row represents a GMG junction. The left panel depicts the perspective view of the optimized structure, the right panel plots the LDOS in the energy window of $\left(E_{\mathrm{f}}-0.5 \mathrm{eV}, E_{\mathrm{f}}+0.5 \mathrm{eV}\right)$, and the bridging molecule is sketched in the middle. The calculated transmission coefficient per spin, $T\left(E_{\mathrm{f}}\right)$, is $0.065(h=7.7 \AA)$ for (a)-(b), $0.014(h=10.3 \AA)$ for (c)-(d), $0.13(h=7.5 \AA)$ for (e)-(f), and $0.068(h=10.96 \AA)$ for $(\mathrm{g})-(\mathrm{h})$, respectively.

channels. The transmission coefficient at Fermi energy, $T\left(E_{\mathrm{f}}\right)$, is proportionally related to the zero-bias conductance, $G$, as $G=2 T\left(E_{\mathrm{f}}\right) g_{0}$ for a spin-closed system with $g_{0}$ being the conductance quantum. For all the four junctions shown in Fig. 2, $T\left(E_{\mathrm{f}}\right)$ is larger than 0.014 , suggesting the ubiquitous applicability of linear conjugated molecules.

In order to reduce the on-bridge scattering to a minimal amount, a suitable conjugated molecule needs to meet the following criteria. (1) It should not be too long since the chance of its getting distorted by external forces increases with length, and any irregular structural change could possibly disturb the $\pi$-conjugation. This is verified by the larger $T\left(E_{\mathrm{f}}\right)$ associated with a dithiophene molecule (OTP2) than that with an OTP3 bridge; see Figs. 2(e)-2(g) and the caption. (2) It should be stiff enough to sustain the stresses induced by suspending the graphene without substantial change in geometry. One counterexample is the OPE2 molecule as depicted in Figs. 2(c) and 2(d). This molecule is easily bent in the middle, leading to the deviation from a coplanar configuration of its two phynel rings, see Fig. 2(c). The off-plane displacements of the two central carbon atoms

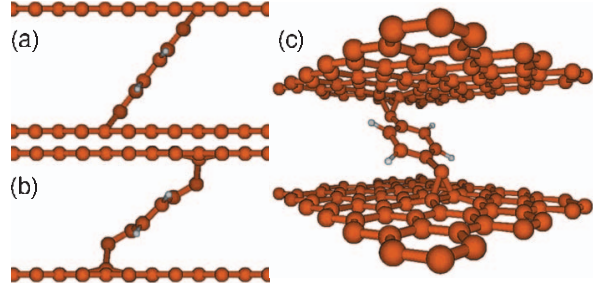

FIG. 3. Side view of the (a) ideal and (b) optimized structures of a graphene-(1,4-diethynylbenzene)-graphene junction. (c) Perspective view from a twisted angle. Note that each link atom is bonded to two adjacent anchor atoms, and forms a three-member ring at each side.

also give rise to the minor gap in the LDOS plotted in Fig. 2(d), which results in the $T\left(E_{\mathrm{f}}\right)$ conspicuously lower than the other bridging molecules under study.

\section{B. Transport transparency of the contact}

To preserve a large conductance for any junction, it is crucial that the conducting channels continue smoothly at the interfaces where different materials or structures meet. For a GMG junction, this requires the link-anchor boundary not to obstruct the extension of $\pi$-conjugation in any way. Ke $e t$ $a l .{ }^{25}$ have studied the contact transparency of nanotubemolecule-nanotube junctions. They have found the sixmember rings at the contact end of a CNT usually break the extension of $\pi$-conjugation; whereas a five-member-ring connection leads to extremely good contact transparency, owing to the in-plane contact geometry which results in almost perfect matching of $\pi$-conjugation at the CNTmolecule interfaces. Apparently, the conductance of GMG junctions would be influenced by the extensity of $\pi$-conjugation in the same fashion.

We now examine how the covalent bonding between the linear molecule and 2D graphene affects the transport properties of a GMG junction. Previous investigations on the chemical functionalization of the CNT walls ${ }^{26,27}$ and 2D graphene ${ }^{11,12}$ have exploited two major bonding schemes: (1) a molecule, such as a phenyl radical, is bound to graphene by a single covalent bond ${ }^{12}$ and (2) a molecule is bounded by two covalent bonds, and forms thus a three-member ring at the contact. ${ }^{12}$ This applies to molecular fragments involving a terminal unsaturated carbon atom. Our studies cover the both schemes related to the GMG junctions, as demonstrated in the left column of Fig. 2.

The $\pi$-conjugation is highly directional. Thus the connection of two conjugated systems in a coplanar (perpendicular) manner is most (least) favorable for the junction conductivity. Consequently, the bridging molecule must always be tilted from an upright position regarding either graphene sheet. Ideally, this would lead to a junction as depicted in Fig. 3(a), where a 1,4-diethynylbenzene (DTB) acts as the bridge, with a three-member ring formed at each contact and coplanar to its phenyl group. However, the contact geometry changes significantly upon optimization, see Fig. 3(b). In particular, the three-member rings are no longer coplanar to the central phenyl ring, but become almost perpendicular to the graphene, see Fig. 3(c). As disclosed in Fig. 4, this leads to a drastic reduction of transmission function near 


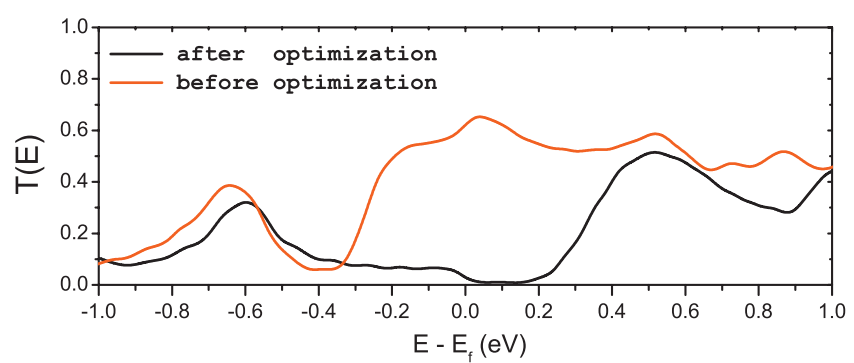

FIG. 4. Calculated zero-bias transmission spectrum for the GMG junction displayed in Figs. 3(a) and 3(b). Note the drastic reduction of $T(\epsilon)$ near $E_{\mathrm{f}}$ upon geometric relaxation.

the Fermi energy. Specifically, $T\left(E_{\mathrm{f}}\right)$ decreases from 0.62 to 0.028 due to the structural modification. While the nearvertical configuration is common for a three-member ring at contact, a single link-anchor bond as joint is found generally less distorted. This suggests that the single-bonding scheme is advantageous in making a conductive GMG junction.

It is thus reasonable to expect the conductance of a GMG junction increases monotonically with the inclination of bridging molecule. To examine this effect, a series of calculations is carried out as follows. First the bridging molecule is tilted to a desired angle. Then the altitudes of bulk graphene sheets are adjusted with respect to the fixed molecular bridge, so that the number of off-plane graphene atoms at contacts reaches global minimum (to be elaborated later). The junction geometry is subsequently optimized with the constrained $h$, based on which the transmission coefficient is finally evaluated. Figure 5 exhibits a GMG junction with a gradually tilted OTP2 bridge obtained through the above procedures. The two parameters adopted to characterize the inclination of the OTP2 bridge are the horizontal distance between the two anchor atoms, $d$; and the vertical separation between the planar graphene layers, $h$. The tilt angle is thus defined as $\theta=\arctan (d / h)$. The corresponding $T\left(E_{\mathrm{f}}\right)$ at various values of $\theta$ are listed in Table I, which verifies the positive correlation between the conductivity and matching of $\pi$-conjugation at the contacts.

Recently Quek et al. ${ }^{28}$ have demonstrated conductance switching in a gold-(4,4'-bipyridine)-gold (Au-BPD-Au)

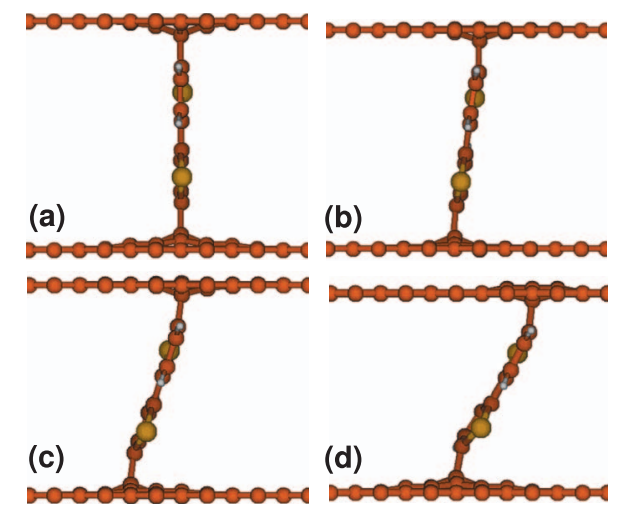

FIG. 5. A GMG junction with a gradually titled OTP2 molecular bridge. The horizontal distance between the two anchor atoms $(d)$ and the vertical separation between the planar areas of graphene $(h)$ are (in unit of $\AA$ ): (a) $d=0.06$ and $h=11.05$, (b) $d=1.23$ and $h=10.6$, (c) $d=2.47$ and $h=10.15$, and (d) $d=3.71$ and $h=9.7$, respectively.
TABLE I. $T\left(E_{\mathrm{f}}\right)$ vs the tilt angle of the OTP2 bridge in a GMG junction depicted in Fig. 4.

\begin{tabular}{cccc}
\hline \hline $\begin{array}{c}d \\
(\AA)\end{array}$ & \multicolumn{1}{c}{$\begin{array}{c}\theta \\
(\AA)\end{array}$} & $\begin{array}{c}\theta \\
(\mathrm{deg})\end{array}$ & $T\left(E_{\mathrm{f}}\right)$ \\
\hline 0.06 & 11.05 & 0.3 & 0.000 \\
1.23 & 10.60 & 6.6 & 0.023 \\
2.47 & 10.15 & 13.7 & 0.029 \\
3.71 & 9.70 & 20.9 & 0.060 \\
4.89 & 9.25 & 27.9 & 0.108 \\
\hline \hline
\end{tabular}

junction by mechanical control of the contact geometry. A similar trend has been observed, i.e., the conductance increases with inclination of bipyridine. This has been attributed to the better matching of the conducting $\pi^{*}$-orbital of bipyridine and lone pair on the (link) nitrogen atom at a larger $\theta$. Despite this analogy, it is worth emphasizing a notable difference between their findings and ours, i.e., for an Au-BPD-Au junction, the preferential orientation of conducting channels inside the bridging molecule is orthogonal to those in the bulk electrodes, while in a GMG junction a parallel matching scenario is favored.

\section{Effects of graphene deformation on conductance}

Consider for instance an OTP2 bridge, which connects to either graphene sheet by a single carbon-carbon bond. To accommodate this extra bond, the anchor atom needs to undergo an $\mathrm{sp}^{2} \rightarrow \mathrm{sp}^{3}$ transformation. This effectively introduces a point defect in the originally perfect $2 \mathrm{D}$ lattice of $\mathrm{sp}^{2}$ carbon atoms, and consequently leads to deformation of graphene in the vicinity of contact. Due to the outstanding in-plane stiffness of graphene that predominates over its flexural rigidity, ${ }^{29}$ and also due to the local curvature induced by the rehybridization of anchor atom, binding with the bridging molecule would primarily result in out-of-plane buckle of the graphene sheet. Such a structural change inevitably modifies the junction conductance and is thus worth exploring.

Although the atomistic details of deformation depend in a complicated way on various factors of the bridging molecule, such as its chemical composition, tilt angle, or binding orientation, the buckle of graphene sheets does exhibit systematic effects on the transport properties of GMG junctions. To exemplify this, below we look into an OTP2-bridged junction with its upper and lower anchor sites separated by a preset horizontal distance $d$. The vertical separation between the two graphene sheets, $h$, is altered consecutively, followed by a geometric optimization at each constrained $h$. This thus mimics a quasistatic compression process, during which the graphene sheets buckle continuously.

From the variation of $T\left(E_{\mathrm{f}}\right)$ with $h$ plotted in Fig. 6 one can see that the transmission coefficient exhibits a continual increase upon compression with rather minor fluctuations. The sustained conductance is due to the slight changes in the atomic positions of bridging molecule and contacts. As an evidence, the vertical distance between the two anchor atoms fluctuates less than $0.2 \AA$ with $h$ ranging from 7.25 to $10 \AA$. Whereas the buckle of graphene, which dominates the structural change, has only secondary effects on the conductance. 


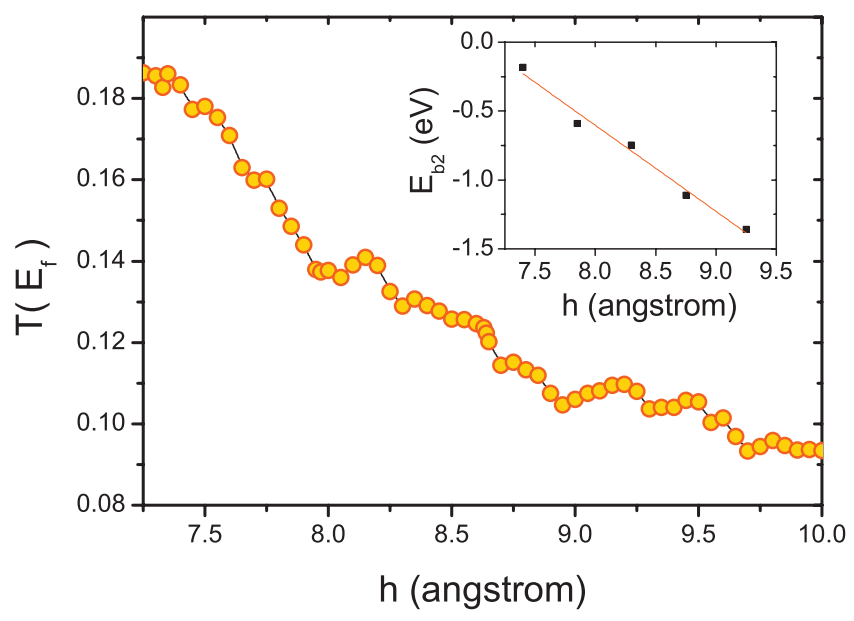

FIG. 6. Transmission coefficient $T\left(E_{\mathrm{f}}\right)$ of a GMG junction of OTP2 molecular bridge as a function of $h$. The circles represent calculated data and the connecting lines are guide to the eyes. The solid squares in inset represent the calculated second binding energy as a function of $h$, and the red line is a linear fit.

The resulting perturbation of the LDOS near around the anchor sites would contribute to the minor fluctuations in $T\left(E_{\mathrm{f}}\right)$ versus $h$. While a more compact junction is energetically unfavorable, it leads to a more pronounced conductance, both due to the enhanced overlap of $\pi$-conjugated electrons. Therefore, a compromise between conductivity and stability needs to be sought in practical design of such GMG junctions. In particular, a zero-bias conductance as large as $0.38 g_{0}$ is reached at $h=7.25 \AA$ (the lower limit of $h$ in the present study). An even larger conductance is expected with further reduced $h$, which however requires extended areas of graphene under explicit optimization.

We now examine the thermal stability of the GMG junctions, which is crucial to the potential applicability of devices based on such junctions. Following the sequential binding scheme proposed in Sec. I, two binding energies are defined, $E_{\mathrm{b} 1} \equiv E(\mathrm{GM} *)-E(\mathrm{G})-E(\mathrm{M} *)$ and $E_{\mathrm{b} 2} \equiv E(\mathrm{GMG})$ $-E(\mathrm{G})-E(\mathrm{GM} *)$, which are associated with the formation of covalent bonds between the bridging molecule and the first and second graphene sheets, respectively. Here, $*$ indicates a radical species when a single anchor-link bond is formed at contact, or a diradical species when a three-member ring is formed. We employ spin-unrestricted DFT calculations to obtained both $E_{\mathrm{b} 1}$ and $E_{\mathrm{b} 2}$, with all involving species at their fully optimized geometries except the vertical separation constraint $h$ imposed on GMG. It is found that $E_{\mathrm{b} 1}$ is negative for all the GMG junctions we have studied. For example, $E_{\mathrm{b} 1}=-2.99 \mathrm{eV}$ for the OTP2 molecular bridge and $-2.59 \mathrm{eV}$ for the DTB bridge, respectively. A negative $E_{\mathrm{b} 1}$ suggests the attachment of the bridging molecule to first graphene sheet is energetically favorable, which is consistent with previous theoretical studies. ${ }^{12}$ In contrast, the second binding energy $E_{\mathrm{b} 2}$ varies with $h$, as the vertical separation between the two graphene sheets in GMG can be tuned by external suspension or pressure. For instance, the explicit $h$-dependence of $E_{\mathrm{b} 2}$ for a G-OTP2-G junction is depicted in the inset of Fig. 6, where the black squares represent the calculated $E_{\mathrm{b} 2}$ at various $h$ ranging from 7.4 to $9.25 \AA$, with the red line representing a linear fit. The overall negative values of $E_{\mathrm{b} 2}$ confirm the thermal stability of the G-OTP2-G junction at $h>7.4 \AA$; while the linear trend suggests that as the two graphene sheets are pushed closer, the fully connected junction would become thermodynamically unstable. The increased $E_{\mathrm{b} 2}$ at a smaller $h$ is largely due to the local buckling of graphene near around the bonded anchor atoms. This confirms that the thermal stability is indeed essential to the applicability of the GMG junction based devices. Combining the $E_{\mathrm{b} 2}$ and $T\left(E_{\mathrm{f}}\right)$ versus $h$ relations as depicted in Fig. 6 , it is interesting to find the conductance of G-OTP2-G junction monitors the variation in geometry and binding stability, as under adjustable external forces. This may lead to potentially useful devices as mechanical/pressure sensors. Moreover, the covalent binding can be further facilitated by modulating the free energy of graphene sheet, $E(\mathrm{G})$, through control of external suspension/pressure or doping of graphene surface. Theoretical studies on phenyl groups binding to a graphene sheet have revealed that the chemisorption of a phenyl radical to graphene sheet is energetically more favorable when the graphene surface is already attached by another phenyl radical. ${ }^{11,12}$

\section{CONCLUDING REMARKS}

To conclude, in this work, we have demonstrated how to bridge two sheets of graphene stacked in parallel in a conductive junction. We have found that the use of a rigid conjugated molecule as the connecting bridge could be a feasible way to establish a conductive junction. To preserve the transport transparency across such a GMG junction, it is of crucial importance to tilt the bridging molecule by a substantial angle, and meanwhile maintain a robust bonding at the bridge-graphene contacts. In doing so, one would achieve a reasonably large conductance, which may have great potential applications in the emerging field of nanoelectronics.

\section{ACKNOWLEDGMENTS}

Support from the Office of Naval Research (Grant No. N00014-09-1-0576) and The National Science Foundation through the funding on Chemical Center of Innovation at Cornell University is gratefully acknowledged.

\section{APPENDIX: SURFACE GREEN FUNCTION FOR A SUPER CELL OF GRAPHENE}

In a one-dimensional quantum transport scenario, there are in general three ways to calculate the surface Green function for an electrode: (1) a highly convergent renormalization scheme ${ }^{30}(2)$ a recursive relation in the form of Dyson equation based on lattice translational invariance; and (3) a wavevector analysis approach. ${ }^{31}$ In contrast, for transport through a GMG junction, the electrodes are 2D graphene sheets. We consider a super cell which consists of a number of unit cells, so that itself is still a repeating unit of the $2 \mathrm{D}$ lattice, and nontrivial interaction exists only between nearest-neighboring super cells. We now try to calculate the surface Green's function associated with this super cell.

The graphene is periodic in 2D real space. Let $\boldsymbol{R}$ be the displacement vector between two super cells, and the home 
super cell is located at $\boldsymbol{R}_{0}$. Consider nonzero overlap integrals between two separated super cells. The surface retarded Green's function, $G_{\boldsymbol{R}_{0}}^{r}\left(\boldsymbol{R}_{1}, \boldsymbol{R}_{2} ; \epsilon\right)$, should satisfy the following equations for all combinations of $\left(\boldsymbol{R}_{1}, \boldsymbol{R}_{2}\right)$ :

$$
\begin{aligned}
& \sum_{\boldsymbol{R}^{\prime}}\left[(\epsilon+i \eta) S_{\boldsymbol{R}^{\prime}}-H_{\boldsymbol{R}^{\prime}}\right] G_{\boldsymbol{R}_{0}}^{r}\left(\boldsymbol{R}^{\prime}, \boldsymbol{R}+\widetilde{\boldsymbol{R}} ; \epsilon\right) \\
& \quad=\delta(\widetilde{\boldsymbol{R}})\left[I-\delta\left(\boldsymbol{R}-\boldsymbol{R}_{0}\right)\right] \\
& \sum_{\boldsymbol{R}^{\prime}} G_{\boldsymbol{R}_{0}}^{r}\left(\boldsymbol{R}+\widetilde{\boldsymbol{R}}, \boldsymbol{R}^{\prime} ; \epsilon\right)\left[(\epsilon+i \eta) S_{\boldsymbol{R}^{\prime} \boldsymbol{R}}-H_{\boldsymbol{R}^{\prime} \boldsymbol{R}}\right] \\
& \quad=\delta(\tilde{\boldsymbol{R}})\left[I-\delta\left(\boldsymbol{R}-\boldsymbol{R}_{0}\right)\right]
\end{aligned}
$$

where $S$ is the overlap matrix, $H$ is the effective singleelectron Hamiltonian matrix, $\delta(\boldsymbol{X})=I$, if $\boldsymbol{X}=\mathbf{0}$ and zero otherwise, and $\eta$ is a positive infinitesimal. The square bracket term on the right-hand side ensures the Green's function vanishes correctly at the boundary of home super cell. Due to the translational invariance of bulk graphene, we denote $S\left(\boldsymbol{R}^{\prime}-\boldsymbol{R}\right) \equiv S_{\boldsymbol{R} \boldsymbol{R}^{\prime}}$ and $H\left(\boldsymbol{R}^{\prime}-\boldsymbol{R}\right) \equiv H_{\boldsymbol{R} \boldsymbol{R}^{\prime}}$. Their counterparts in the reciprocal space are

$$
\begin{aligned}
& S(\boldsymbol{K})=\sum_{\boldsymbol{R}} e^{i \boldsymbol{K} \cdot \boldsymbol{R}} S(\boldsymbol{R}), \\
& H(\boldsymbol{K})=\sum_{\boldsymbol{R}} e^{i \boldsymbol{K} \cdot \boldsymbol{R}} H(\boldsymbol{R}) .
\end{aligned}
$$

The retarded bulk Green's function in reciprocal space is $G_{0}^{r}(\boldsymbol{K} ; \epsilon)=[(\epsilon+i \eta) S(\boldsymbol{K})-H(\boldsymbol{K})]^{-1}$. The real-space Green's function corresponds to an inverse Fourier transform of

$$
G_{0}^{r}(\boldsymbol{R} ; \boldsymbol{\epsilon})=\int d \boldsymbol{K} G_{0}^{r}(\boldsymbol{K} ; \boldsymbol{\epsilon}) e^{-i \boldsymbol{K} \cdot \boldsymbol{R}} .
$$

Obviously, it depends only on the displacement vector between two super cells due to the translational invariance.

As the effective single-electron Hamiltonian matrix of home super cell, $H_{\boldsymbol{R}_{0} \boldsymbol{R}_{0}}$, is modified by binding to the bridging molecule, and deviates from the bulk Hamiltonian, an additional surface term, $\Delta G_{\boldsymbol{R}_{0}}\left(\boldsymbol{R}_{1}, \boldsymbol{R}_{2} ; \epsilon\right)$, is needed to account for the vanishing amplitude of Green function at the boundary of home super cell. This surface term thus breaks the translational invariance of $\boldsymbol{R}_{2}-\boldsymbol{R}_{1}$. In this case, the home super cell mimics a "point defect" in a perfect lattice. Altogether we have

$$
\begin{aligned}
G_{\boldsymbol{R}_{0}}^{r}\left(\boldsymbol{R}_{1}, \boldsymbol{R}_{2} ; \epsilon\right)= & G_{0}^{r}\left(\boldsymbol{R}_{2}-\boldsymbol{R}_{1} ; \epsilon\right)-\Delta G_{\boldsymbol{R}_{0}}\left(\boldsymbol{R}_{1}, \boldsymbol{R}_{2} ; \epsilon\right), \\
\Delta G_{\boldsymbol{R}_{0}}\left(\boldsymbol{R}_{1}, \boldsymbol{R}_{2} ; \epsilon\right)= & G_{0}^{r}\left(\boldsymbol{R}_{0}-\boldsymbol{R}_{1} ; \epsilon\right)\left[G_{0}^{r}(\mathbf{0} ; \epsilon)\right]^{-1} \\
& \times G_{0}^{r}\left(\boldsymbol{R}_{2}-\boldsymbol{R}_{0} ; \epsilon\right),
\end{aligned}
$$

which satisfy Eq. (A1). It remains trivial to verify the formal equivalence of $\Delta G_{\boldsymbol{R}_{0}}\left(\boldsymbol{R}_{0}, \boldsymbol{R}_{0} ; \epsilon\right)=G_{0}^{r}(\mathbf{0} ; \epsilon)$, and it is also apparent that $G_{\boldsymbol{R}_{0}}^{r}\left(\boldsymbol{R}_{1}, \boldsymbol{R}_{0} ; \epsilon\right)=G_{\boldsymbol{R}_{0}}^{r}\left(\boldsymbol{R}_{0}, \boldsymbol{R}_{2} ; \epsilon\right)=0$. The boundary conditions are thus accounted for properly.

In practice, The $\boldsymbol{K}$-space integration involved in Eq. (A3) needs to be evaluated explicitly, which requires a careful sampling of the Brillouin zone. A $\boldsymbol{\Gamma}$-point approximation would be the worst estimation for $G_{\boldsymbol{R}_{0}}^{r}$ since it gives rise to
$G_{\boldsymbol{R}_{0}}^{r}\left(\boldsymbol{R}_{1}, \boldsymbol{R}_{2} ; \epsilon\right)=0, \forall\left(\boldsymbol{R}_{1}, \boldsymbol{R}_{2}\right)$. With the surface Green function, the retarded self-energy required in our DFT-NEGF calculation, $\sum_{\boldsymbol{R}_{0} \boldsymbol{R}_{0}}^{r}(\epsilon)$, is obtained via

$$
\begin{aligned}
\sum_{\boldsymbol{R}_{0} \boldsymbol{R}_{0}}^{r}(\epsilon)= & \sum_{\boldsymbol{R}_{1} \neq \boldsymbol{R}_{0}} \sum_{\boldsymbol{R}_{2} \neq \boldsymbol{R}_{0}} \tilde{H}_{\boldsymbol{R}_{0} \boldsymbol{R}_{1}}(\epsilon) G_{\boldsymbol{R}_{0}}^{r}\left(\boldsymbol{R}_{1}, \boldsymbol{R}_{2} ; \epsilon\right) \tilde{H}_{\boldsymbol{R}_{2} \boldsymbol{R}_{0}}(\epsilon) \\
= & \sum_{\boldsymbol{R}_{2}>\boldsymbol{R}_{1}}\left[\widetilde{H}_{\boldsymbol{R}_{0} \boldsymbol{R}_{1}} G_{\boldsymbol{R}_{2}-\boldsymbol{R}_{1}}^{0} \tilde{H}_{\boldsymbol{R}_{2} \boldsymbol{R}_{0}}+\tilde{H}_{\boldsymbol{R}_{0} \boldsymbol{R}_{2}} G_{\boldsymbol{R}_{1}-\boldsymbol{R}_{2}}^{0} \tilde{H}_{\boldsymbol{R}_{1} \boldsymbol{R}_{0}}\right] \\
& +\sum_{\boldsymbol{R}_{1} \neq \boldsymbol{R}_{0}} \tilde{H}_{\boldsymbol{R}_{0} \boldsymbol{R}_{1}} G_{0}^{0} \tilde{H}_{\boldsymbol{R}_{1} \boldsymbol{R}_{0}} \\
& +\left[\sum_{\boldsymbol{R}_{1} \neq \boldsymbol{R}_{0}} \tilde{H}_{\boldsymbol{R}_{0} \boldsymbol{R}_{1}} G_{-\boldsymbol{R}_{1}}^{0}\right] \tilde{H}_{\boldsymbol{R}_{0} \boldsymbol{R}_{0}}\left[\sum_{\boldsymbol{R}_{1} \neq \boldsymbol{R}_{0}} G_{\boldsymbol{R}_{1}}^{0} \tilde{H}_{\boldsymbol{R}_{1} \boldsymbol{R}_{0}}\right] .
\end{aligned}
$$

Here, $\tilde{H}_{\boldsymbol{R}_{0} \boldsymbol{R}_{1}}(\epsilon) \equiv(\epsilon+i \eta) S_{\boldsymbol{R}_{0} \boldsymbol{R}_{1}}-H_{\boldsymbol{R}_{0} \boldsymbol{R}_{1}}$, and $G_{\boldsymbol{R}_{1}}^{0} \equiv G_{0}^{r}\left(\boldsymbol{R}_{1} ; \epsilon\right)$.

To accurately evaluate the zero-bias conductance of the GMG junctions, a super cell consisting of $72(12 \times 6)$ carbon atoms is adopted for each graphene sheet. A set of $(25 \times 25$ $\times 1)$ Monkhorst-Pack grids ${ }^{32}$ is established for the $\boldsymbol{K}$-space sampling. A relatively large $\eta=0.04 \mathrm{eV}$ is used as the broadening of graphene states. In principle, $\eta$ should be infinitesimally small. However, with a much smaller $\eta$, the reciprocal space sampling would converge extremely slowly by increasing the density of $\boldsymbol{K}$-grids; while a too large $\boldsymbol{\eta}$ may artificially lower and broaden the peaks in the transmission spectrum, $T(\epsilon)$.

${ }^{1}$ K. S. Novoselov, A. K. Geim, S. V. Morozov, D. Jiang, Y. Zhang, S. V. Dubonos, I. V. Grigorieva, and A. A. Firsov, Science 306, 666 (2004).

${ }^{2}$ A. K. Geim and K. S. Novoselov, Nature Mater. 6, 183 (2007).

${ }^{3}$ K. S. Novoselov, D. Jiang, F. Schedin, T. J. Booth, V. V. Khotkevich, S. V. Morozov, and A. K. Geim, Proc. Natl. Acad. Sci. U.S.A. 102, 10451 (2005).

${ }^{4}$ L. A. Ponomarenko, F. Schedin, M. I. Katsnelson, R. Yang, E. W. Hill, K. S. Novoselov, and A. K. Geim, Science 320, 356 (2008).

${ }^{5}$ F. Schedin, A. K. Geim, S. V. Morozov, E. W. Hill, P. Blake, M. I. Katsnelson, and K. S. Novoselov, Nature Mater. 6, 652 (2007).

${ }^{6}$ E. W. Hill, A. K. Geim, K. Novoselov, F. Schedin, and P. Blake, IEEE Trans. Magn. 42, 2694 (2006).

${ }^{7}$ J. S. Bunch, S. S. Verbridge, J. S. Alden, A. M. van der Zande, J. M. Parpia, H. G. Craighead, and P. L. McEuen, Nano Lett. 8, 2458 (2008).

${ }^{8}$ M. Delamar, R. Hitmi, J. Pinson, and J. M. Saveant, J. Am. Chem. Soc. 114, 5883 (1992).

${ }^{9}$ J. L. Bahr and J. M. Tour, J. Mater. Chem. 12, 1952 (2002).

${ }^{10}$ M. S. Strano, C. A. Dyke, M. L. Usrey, P. W. Barone, M. J. Allen, H. Shan, C. Kittrell, R. H. Hauge, J. M. Tour, and R. E. Smalley, Science 301, 1519 (2003)

${ }^{11}$ D. E. Jiang, B. G. Sumpter, and S. Dai, J. Phys. Chem. B 110, 23628 (2006).

${ }^{12}$ E. R. Margine, M. L. Bocquet, and X. Blase, Nano Lett. 8, 3315 (2008).

${ }^{13}$ J. M. Soler, E. Artacho, J. D. Gale, A. Garcia, J. Junquera, P. Ordejon, and D. Sanchez-Portal, J. Phys.: Condens. Matter 14, 2745 (2002).

${ }^{14}$ L. V. Keldysh, Zh. Eksp. Teor. Fiz. 47, 1515 (1964).

${ }^{15}$ W. Kohn and L. J. Sham, Phys. Rev. 140, A1133 (1965).

${ }^{16}$ S. H. Ke, H. U. Baranger, and W. T. Yang, Phys. Rev. B 70, 085410 (2004).

${ }^{17}$ F. Evers, F. Weigend, and M. Koentopp, Phys. Rev. B 69, 235411 (2004).

${ }^{18}$ C. Toher, A. Filippetti, S. Sanvito, and K. Burke, Phys. Rev. Lett. 95, 146402 (2005).

${ }^{19}$ S. H. Ke, H. U. Baranger, and W. T. Yang, J. Chem. Phys. 126, 201102 (2007).

${ }^{20}$ P. Mori-Sánchez, A. J. Cohen, and W. T. Yang, Phys. Rev. Lett. 100, 146401 (2008).

${ }^{21}$ A. J. Cohen, P. Mori-Sánchez, and W. T. Yang, Science 321, 792 (2008).

${ }^{22}$ R. Landauer, Philos. Mag. 21, 863 (1970).

${ }^{23}$ M. Büttiker, Y. Imry, R. Landauer, and S. Pinhas, Phys. Rev. B 31, 6207 
(1985).

${ }^{24}$ S. Datta, Electronic Transport in Mesoscopic Systems (Oxford University Press, New York, 1995).

${ }^{25}$ S. H. Ke, H. U. Baranger, and W. T. Yang, Phys. Rev. Lett. 99, 146802 (2007)

${ }^{26}$ H. Park, J. J. Zhao, and J. P. Lu, Nano Lett. 6, 916 (2006).

${ }^{27}$ A. López-Bezanilla, F. Triozon, S. Latil, X. Blase, and S. Roche, Nano Lett. 9, 940 (2009).

${ }^{28}$ S. Y. Quek, M. Kamenetska, M. L. Steigerwald, H. J. Choi, S. G. Louie,
M. S. Hybertsen, J. B. Neaton, and L. Venkataraman, Nat. Nanotechnol. 4, 230 (2009).

${ }^{29}$ K. N. Kudin, G. E. Scuseria, and B. I. Yakobson, Phys. Rev. B 64, 235406 (2001).

${ }^{30}$ M. P. L. Sancho, J. M. L. Sancho, and J. Rubio, J. Phys. F: Met. Phys. 15, 851 (1985).

${ }^{31}$ S. Sanvito, C. J. Lambert, J. H. Jefferson, and A. M. Bratkovsky, Phys. Rev. B 59, 11936 (1999).

${ }^{32}$ H. J. Monkhorst and J. D. Pack, Phys. Rev. B 13, 5188 (1976). 